\title{
Comments on "Noninvasive Brain Stimulation for Poststroke Dysphagia: A Meta-Analysis for Randomized Controlled Trials" by Zhu et al.
}

\author{
Seoyon Yang ${ }^{a}$ Min Cheol Chang ${ }^{b}$ \\ aDepartment of Rehabilitation Medicine, Ewha Womans University Seoul Hospital, Ewha Womans University \\ School of Medicine, Seoul, South Korea; 'bepartment of Rehabilitation Medicine, College of Medicine, Yeungnam \\ University, Daegu, South Korea
}

Dear Editor,

We read the recently published article "Noninvasive Brain Stimulation for Poststroke Dysphagia: A MetaAnalysis for Randomized Controlled Trials" by Zhu and $\mathrm{Gu}$ [1] with great interest. This was a meta-analysis of seven articles that evaluated the effectiveness of repetitive transcranial magnetic stimulation (rTMS) or transcranial direct current stimulation (tDCS) for managing poststroke dysphagia. As the authors described in their article, the effectiveness of rTMS and tDCS for post-stroke dysphagia remains controversial, therefore warranting a meta-analysis. However, there are some important issues.

First, different outcome measurements were analyzed together for the meta-analysis. A variety of tools or scores were used to assess improvements in the severity of dysphagia in the included studies: functional oral intake scale, videofluoroscopic swallowing studies, videofluoroscopic dysphagia scale, penetration-aspiration scale, dysphagia outcome and severity scale, functional dysphagia scale, and standardized swallowing assessment, swallowing-related quality of life questionnaire, and maximal tongue strength measurement. Each study included in the meta-analysis used only a few of the abovementioned measurement tools. Therefore, the measurement tools used in the studies included in Zhu and Gu meta-analysis were different for each study. For the accurate evaluation of the effectiveness of rTMS and tDCS on post-stroke dysphagia, outcome measurements in each study should have been classified according to the type of evaluation methods and analyzed separately. Second, a study (Kim et al.) included in this meta-analysis recruited patients with traumatic brain injury (TBI) and performed rTMS on them [2]. Because this meta-analysis aimed to evaluate the effect of rTMS and tDCS on post-stroke dysphagia, Zhu et al. should have controlled or deleted the outcomes of patients with TBI.

In Zhu and Gu meta-analysis, the standardized mean differences for rTMS and tDCS on post-stroke dysphagia were 1.08 and 1.43, respectively. Based on Cohen's study, these effect size values can be interpreted as rTMS and tDCS having a large positive therapeutic effect on poststroke dysphagia [3]. However, due to the aforementioned issues, we believe that the results of Zhu et al.'s study may not be reliable.

\section{Conflict of Interest Statement}

The authors declare no conflict of interest.
Karger@karger.com www.karger.com/ene
(C) 2022 S. Karger AG, Basel

Karger
Correspondence to:

Min Cheol Chang,wheel633@gmail.com 


\section{Funding Sources}

We received no funding for this study.

\section{Author Contributions}

S.Y. and M.C.C.: concept and study design, writing of manuscript, critical editing of the manuscript. M.C.C.: supervised the study. All authors have read and agreed to the published version of the manuscript.
1 Zhu Y, Gu L. Noninvasive brain stimulation for poststroke dysphagia: a meta-analysis for randomized controlled trials. Eur Neurol. 2021:1-8. Online ahead of print.

2 Kim L, Chun MH, Kim BR, Lee SJ. Effect of repetitive transcranial magnetic stimulation on patients with brain injury and dysphagia. Ann Rehabil Med. 2011;35(6):765-71.

3 Cohen J. Statistical power analysis for the behavioral sciences. 2nd ed. Hillsdale, NJ, USA: Erlbaum; 1988. 